\title{
Adenocarcinoma de cérvix
}

\author{
Hernán Urdaneta Tovar, MD*
}

\begin{abstract}
RESUMEN: Se presenta una revisión del Adenocarcinoma de cérvix en sus aspectos epidemiológicos, diagnóstico en estados inicialés y colposcópicos.
\end{abstract}

PALABRAS CLAVES: Adenocarcinoma del cérvix, atipias glandulares de significado incierto, adenocarcinoma In Situ, colposcópicos.

SUMMARY: It's done a revision of the Adenocarcinoma of the cervix in epidemic aspects, diagnosis in initial stages and colposcopic aspects.

KEY WORDS: Adenocarcinoma of the cervix, glandular atipia, uncaertain significance, In Situ Adenocarcinoma, colposcopy.

\section{Incidencia \\ Se ha reportado un amplio rango en la frecuencia de Adenocarcinoma de cérvix: $2-30 \%$ del total de cánceres de cérvix, en promedio 8-20\% en la actualidad. Este amplio rango refleja la dificultad de diferenciar en el examen histológico el adenocarcinoma del carcinoma escamocelular de cérvix. Su incidencia ha aumentado en un 4\% en mujeres menores de 35 años a partir de 1965 . Es más frecuente en EE.UU. y Europa que en Japón .}

\section{Factores de riesgo}

Al parecer se pueden identificar dos subgrupos de adenocarcinomas de cérvix: uno con factores de riesgo similares a los del adenocarcinoma de endometrio (nulíparas o con pocos embarazos, más ancianas, más frecuentemente obesas y diabéticas) y otro con factores de riesgo más semejantes a los del carcinoma escamocelular de cérvix (multiparidad, infección por VPH en especial el tipo 18).

El VPH se detecta en el $80 \%$ de los adenocarcinomas infiltrantes, en el 40-70\% de los adenocarcinomas de cérvix in situ (ACIS) y en unas pocas displasias glandulares de bajo grado. Algunas de éstas anormalidades glandulares de bajo grado pueden ser debidas a condiciones inflamatorias más que a procesos neoplásicos.

\section{Histología}

Los tipos histológicos corresponden a:

Adenocarcinoma verdadero

Adenoma maligno
Profesor Asociado. Unidad de Patología del Tracto Genital Inferior y Colposcopia. Departamento de Ginecología y Obstetricia. Hospital Militar Central.

\author{
Mucinoso \\ Papilar \\ Endometrioide \\ De células claras \\ Adenoide quístico \\ Existe también el Carcinoma Adenoescamoso
}

\section{Diagnóstico citológico}

La identificación de la displasia glandular y del Adenocarcinoma in Situ (ACIS) y su tratamiento tendrían las mismas ventajas que la identificación y tratamiento de la Neoplasia Intraepitelial Cervical (NIC) que han determinado con relación al carcinoma escamoso.

Desafortunadamente en el Adenocarcinoma de Cérvix la citología presenta aun menor confiabilidad diagnóstica que la observada en el carcinoma Escamocelular: los falsos negativos de la citología eñè Carcinoma Escamoso Infiltrante son del orden del 20\% mientras que en el Adenocarcinoma Invasor alcanzan el $40 \%$. La sensibilidad disminuida de la citología puede ser debida a una mayor irregularidad en el desprendimiento de las células glandulares anormales, pequeño tamaño de las lesiones y localización endocervical. Adicionalmente la citología correspondiente a un adenocarcinoma bien diferenciado temprano puede ser sutilmente diferente de lo normal.

El Sistema de Bethesda creó el términoAGUS(Atypical Glandular Cells of Undetermined Significance o Atipias Celulares Glandulares de Significado Incierto), aplicable cuando los cambios celulares glandulares exceden a los esperados en una reacción reactiva o reparativa pero no son aún claramente neoplásicos. A pesar del potencial etiológico benigno de AGUS, esta categoría presenta un mayor riesgo que el propio de ASCUS (Atypical Squamous Cells of Undetermined Significance), ya que entre el 30 al $50 \%$ de las mujeres con diagnóstico citológico de AGUS presentan displasia, ACIS o cáncer. 
En la Clasificación de Papanicolau muchos patólogos emplean el término "Atipias Severas" aplicando el mismo concepto que se usa para definir los términos ASCUS y AGUS.

Se ha informado que en el $43 \%$ de casos de ACIS coexisten simultáneamente con NIC, lo que sugiere que el mecanismo histogénico del Adenocarcinoma Cervical sería el mismo del Carcinoma Escamoso, y que las células de origen serían las Células de Reserva subcilíndricas.

Las atipias glandulares preinvasivas no sólo son difíciles de identificar mediante citología sino también existe gran dificultad colposcópica. Aunque pueden afectar cualquier área del epitelio glandular columnar, comúnmente se desarrollan cerca de la unión escamocolumnar. Las lesiones pueden ser focales, multicéntricas o difusas.

Ciertamente, toda mujer que presente AGUS, debe practicársele como mínimo colposcopia del Cérvix, colposcopia vaginal completa y curetaje endocervical. No hay que olvidar que las células glandulares anormales pueden originarse en el Cérvix (porción visible o en el canal), vagina, endometrio, trompas, ovarios y metástasis (gastrointestinal, senos). Las células que parecen células endocervicales anormales no siempre son de origen endocervical ya que pueden ser metaplásicas o reflejar una lesión escamosa. La confirmación por un segundo citopatólogo siempre es deseable atendiendo la dificultad que existe para el diagnóstico.

El ACIS se diagnostica con menor frecuencia que el adenocarcinoma reflejando quizá la dificultad tanto citológica como colposcópica para identificar las lesiones precursoras así como la posible progresión rápida de tales lesiones. Realmente el ACIS y el adenocarcinoma no pueden diferenciarse entre sí con base en la citología únicamente, su diagnóstico requiere demostrar cambios histológicos.

Toda mujer con ACIS sospechado por citología o por Bx debe ser evaluada mediante conización para definir así la extensión de la lesión así como excluir la presencia de invasión.

\section{Manejo del ASGUS - ASCUS}

1- Colposcopia para todas las mujeres con ASCUS (AGUS está asociado con alto riesgo de NIC, especialmente de Alto Grado, asociado también con ASGUS y adenocarcinoma).

2- Curetaje endocervical para todas las mujeres con AGUS, aún si el canal endocervical presenta apariencia normal.

3- Evaluación colposcópica de toda la vagina.

Si tanto el examen colposcópico como el legrado endocervical son negativos, hasta no conocer mejor la historia natural de las lesiones glandulares, parece prudente realizar controles citológicos cada 4 meses hasta obtener un mínimo de 4 extendidos citológicos negativos.

Si la biopsia (Bx) y/o el curetaje endocervical son positivos para Neoplasia (excluyendo cáncer infiltrante), debe realizarse cono o escisión electroquirúrgica. Debido a los artefactos térmicos que acompañan este último procedimiento, no pocos médicos se inclinan más por la práctica de conización en frío o con láser. El cono debe ser profundo $(2.0-2.5 \mathrm{~cm})$ y debe incluir en su totalidad la zona de transformación.

\section{Manejo de la sospecha de ASGIS o ASGUS de Adeno- carcinoma}

El cáncer cervical glandular puede ser rápidamente progresivo.

1- Toda mujer con diagnóstico citológico sugestivo o sospechoso de ASGIS o de Adenocarcinoma, debe practicarse colposcopia, $\mathrm{Bx}$ dirigidas y legrado endocervical.

2- Si procediendo como en el punto anterior no se localiza el sitio de la anormalidad, debe evaluarse la vagina mediante colposcopia, coloración de Lugol y realizarse palpación cervicovaginal. Si aún así no se encuentra lesión, el extendido citológico debe ser revisado por un segundo citopatólogo. Este paso es de particular importancia, ya que una inflamación endocervical severa, la hiperplasia microglandular endocervical, la metaplasia mulleriana incluyendo los tipos celulares ciliados y endometriales, el fenómeno de Arias-Stella, la proliferación adenomatoide y remanentes mesonéfricos pueden todos remedar ASGUS o adenocarcinoma.

3- Si el diagnóstico citológico es concluyente de ACIS o de adenocarcinoma, debe practicarse cono.

\section{Clasificación por Estadios Adenocarcinoma de Cérvix}

La clasificación por estadios del Adenocarcinoma de Cérvix es análoga a la del carcinoma escamocelular.

Las etapas se definen por clasificación FIGO o TNM.

\section{Aspectos colposcópicos del adenocarcinoma de cérvix}

- Observación directa: Color, lustre, márgenes, forma de glándulas abiertas y la apariencia de los vasos sanguíneos.

- Observación después de la aplicación de ácido acético al $5 \%$.

Importa mucho el color (diferentes gradaciones de amarillo) y el tamaño y la forma de los orificios glandulares. Igual el patrón vascular, especialmente los vasos gruesos parecidos a raíces (Root-like) o los capilares finos que semejan fragmentos, gusanitos, desperdicios o desechos de hilo o lanitas (Waste-thread like).

El Adenocarcinoma Infiltrante es posible diagnosticarlo por colposcopia, mientras que el adenocarcinoma in situ suele pasar inadvertido.

Los hallazgos colposcópicos del adenocarcinoma difieren considerablemente de aquellos del epitelio escamoso anormal. Este último incluye mosaico, punteado, epitelio acetoblanco y keratosis, que si bien se encuentran en el Carcinoma Adenoescamoso, nunca se ven en el Adenocarcinoma Verdadero. Esto se debe a que, a diferencia del Carcinoma Escamoso que se origina en cambios de la zona de transformación, vale decir, los hallazgos anormales del Carcinoma Escamoso son parte de la zona de transformación anormal, el Adenocarcinoma raramente se desarrolla en la zona de transformación como resultado de metaplasia epitelial, por lo que el curso de las anormalidades del epitelio glandular no se parecen a las del epitelio escamoso. 
Existen seis hallazgos colposcópicos que pueden ser característicos o acompañar el Adenocarcinoma Cervical.

\section{Hallazgos papilares $(\mathbf{1 7 \%})$}

La apariencia es similar a la del epitelio columnar, sin embargo sin ácido acético la superficie papilar hemorrágica es de color amarillo pálido claramente delimitada. La cantidad de moco puede ser normal (Adenocarcinoma típico) o copiosa (Adenocarcinoma Secretante de Moco). Al acético la superficie se torna de color blanco lechoso moderado, cada papila es grande sin uniformidad en el tamaño. Esparcidas en la superficie papilar se encuentran equitativamente orificios glandulares grandes en el caso del tipo de adenocarcinoma muco-secretante, pero raramente en el adeno típico. Cada papila presenta vasos glomeruloides o pueden ser en horquilla de pelo, y en las papilas grandes, los vasos son semejantes a raíces.

Los adenocarcinomas típicos avanzados poseen superficies hemorrágicas, destrucción temprana de la superficie, o costra necrótica. Estos hallazgos casi nunca los presenta el tipo muco-secretante.

2. Hallazgos semejantes a Zona de Transformación (ZT). (9\%)

El Cérvix parece presentar una zona de transformación pero esta es gruesa, sin brillo, color naranja pálido y con amplia variedad de patrones vasculares: Parecidos a raíces (Root-like), semejantes a desechos de lanas o gusanitos (Waste thread-like), en asa (Loop-like), en rama de sauce (willow branch-like) y de tipo linear.

Después de la aplicación del acético la superficie se torna blanco lechosa, los orificios glandulares son en general grandes e irregularmente diseminados. No se observan círculos blancos alrededor de los orificios glandulares abiertos. En los adenos mucosecretantes, los orificios son más grandes y la producción de moco mayor.

\section{Hallazgos de vasos Atípicos (3\%)}

Sin acético se observa un área amarillenta sin orificios glandulares visibles, con vasos semejantes a raíces, sacacorchos (corkscrew-like), desechos de lanas y zarcillos (tendril-like) después de la aplicación de acético se observa un blanqueamiento moderado y los orificios glandulares se tornan visibles.

El patrón vascular más frecuente en casos de adenocarcinoma es el de vasos semejantes a raíces. En los estadios tempranos son visibles uno o dos de tales vasos, pero en los estadios avanzados son numerosos. En los estadios iniciales son visibles vasos tipo lanitas mientras que en los estadios avanzados se incrementan los zarcillos y las ramas de sauce aparecen.

Es de gran interés la rareza de los vasos en sacacorchos que son vistos frecuentemente en el carcinoma escamocelular.

Los vasos semejantes a raíces son característicos del adenocarcinoma papilar, mientras que los lanitas son vistos en el adenocarcinoma tipo Zona de Transformación, en el tipo de vasos atípicos, en el tipo granulación y en los adenocarcinomas sin patrón definido. En los casos avanzados las raíces, las ramas de sauce, las lanitas o gusanitos y los zarcillos son numerosos.

\section{Hallazgos semejantes a Granulación (4\%)}

En el adeno típico el área semejante a tejido de granulación está levantada, la superficie es irregular, amarilla y contiene vasos semejantes a raíces, desechos de lanitas, sacacorchos, y glomeruloides, a menudo congestionados, sin observarse orificios glandulares, después de la aplicación de acético se produce un ligero blanqueamiento y empalidecimiento del amarillo. Los casos avanzados muestran hemorragia, necrosis, superficie costrosa que no están presentes en los mucosecretantes.

\section{Hallazgos indistintos $(2 \%)$}

Sólo se observa en el adeno muco-secretante. El Cérvix se observa agrandado, enrojecido, con frecuencia erosionado ampliamente y cubierto con un exudado mucoso copioso. La superficie es roja opaca entrelazada dando una apariencia homogénea. El acético causa un blanqueamiento discreto y resalta la apariencia no característica. Los vasos están congestivos lo que dificulta establecer cualquier patrón. En los intersticios se observan algunas papilas y orificios glandulares grandes.

\section{6 . Hallazgos en casos avanzados $(56 \%)$}

Hemorragia, necrosis, destrucción temprana de la superficie, costras necróticas.

Los hallazgos tempranos Papilares y semejantes a Zona de Transformación se presentan conjuntamente en un $9 \%$.

\section{Examen de los vasos cervicales}

La apariencia de los vasos sanguíneos en colposcopia tiene significado diagnóstico importante, pero más aún en los casos de adenocarcinoma. Ya que la aplicación del ácido acético los oculta, los vasos deben ser examinados antes de exponer el Cérvix al acético. Puede ser usado un filtro verde o la aplicación de noradrenalina, pero la simple observación con frecuencia es suficiente y con la práctica fácil.

Existen 13 tipos de vasos sanguíneos, 6 de los cuales (típicos) son visibles en epitelio no maligno y 7 (atípicos) en neoplasias cervicales. Como quiera que sea, en adición a los vasos atípicos pueden observarse patrones vasculares normales.

A menudo, entre más avanzada sea la Neoplasia mayor variedad de tamaños y distribución de los vasos.

Por supuesto que existen algunos casos de adenocarcinoma avanzado en que los vasos sanguíneos no muestran anormalidades como las aquí descritas.

Los tipos de Vasos sanguíneos normales (VN) o típicos son:

- Retículo, malla o Red (VN 1)

- Moteado Rojo o Hemorragias intraparenquimatosas (VN 2)

- Punteado regular fino (VN 3)

- Ramificados semejantes a árbol (VN 4)

- Lineares (VN 5)

- En asa (VN 6)

Los vasos atípicos o anormales (VA) son:

- Glomeruloides y en Horquilla (VA 1)

- Sacacorchos (VA 2)

- Mosaico (VA 3) 
Figura

DIAGRAMA DEL PATRON VASCULAR CERVICAL

VN: VASOS NORMALES - VA: VASOS ANORMALES

\begin{tabular}{|c|c|c|c|c|c|}
\hline $\begin{array}{c}\text { RETICULO } \\
\text { VN I }\end{array}$ & $\begin{array}{l}\text { MOTEADO } \\
\text { ROJO } \\
\text { VN } 2\end{array}$ & $\begin{array}{c}\text { PUNTEADO } \\
\text { FINO } \\
\text { VN } 3 \\
\end{array}$ & $\begin{array}{c}\text { RAMIFICADOS } \\
\text { VN } 4\end{array}$ & $\begin{array}{l}\text { LINEAR } \\
\text { VN } 5\end{array}$ & $\begin{array}{l}\text { EN ASA } \\
\text { VN } 6 \\
\end{array}$ \\
\hline 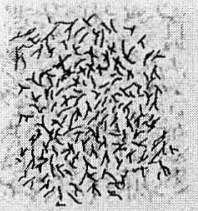 & $\begin{array}{l}0 \\
0\end{array}$ & 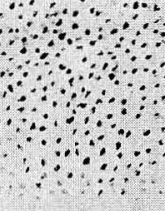 & तनीद & $131\}$ & $\begin{array}{l}49 \\
90 \\
90 \\
0\end{array}$ \\
\hline
\end{tabular}

\begin{tabular}{|c|c|c|c|c|c|c|}
\hline $\begin{array}{c}\text { GLOMERULOIDES } \\
\text { ORQUILLA } \\
\text { VA I }\end{array}$ & $\begin{array}{c}\text { SACACORCHOS } \\
\text { VA } 2\end{array}$ & MOSAICO & ZARC̣LLOS & $\begin{array}{l}\text { GUSANITOS } \\
\text { DESPERDICIOS } \\
\text { DE LANAS } \\
\text { VA5 }\end{array}$ & $\begin{array}{c}\text { RAMAS } \\
\text { DE SAUCE } \\
\text { VA } 6 \\
\end{array}$ & $\begin{array}{l}\text { RAICES } \\
\text { VA } 7 \\
\end{array}$ \\
\hline 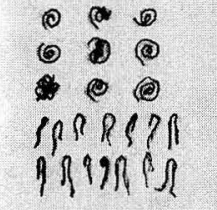 & 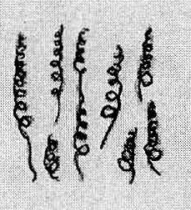 & & & & $(4(5)(5)\}$ & \\
\hline
\end{tabular}

- Zarcillo (VA 4)

- Desperdicios de lanitas o gusanitos (VA 5)

- Rama de sauce (VA 6)

- Raíces (VA 7)

\section{Hallazgos colposcópicos en adenocarcinoma temprano}

La displasia glandular (Concepto histológico no aceptado de manera universal) y el adenocarcinoma in situ con frecuencia son lesiones muy pequeñas. Ya que se encuentran por debajo de la superficie, no pueden ser vistas por colposcopia lo mismo que el adenocarcinoma invasivo muy temprano.

\section{Hallazgos colposcópicos en adenocarcinoma verdadero} (Secretante de cantidades normales de moco)

La superficie suele presentar alguna erosión cuando se trata de casos tempranos. Con casos más avanzados, el Cérvix puede encontrarse hinchado o con áreas de depresión.

La superficie erodada es amarillenta y muchas veces nublada.

Los hallazgos colposcópicos en el adenocarcinoma estadio 1 son:

Estructuras papilares (29.4\%), parecido a la Zona de Transformación (ZT) (17.6\%), combinación de hallazgos papilares y hallazgos de ZT (29.4\%), semejante a tejido de granulación $(0 \%)$ y vasos atípicos $(11.8 \%)$. En estadios 2 la superficie se observa destruida en el $67 \%$ de los casos por lo que los porcentajes de hallazgos papilares, de ZT y combinación de ambos disminuyen proporcionalmente. Los hallazgos de granulación son del orden del 5.8\%.

En el adenocarcinoma verdadero, estadios 1 y 2 , los hallazgos característicos de la ZT atípica no se observan como mosaico, punteado, epitelio blanco o keratosis.

\section{Manifestaciones papilares}

Las papilas observadas pueden ser de tamaño uniforme pero algo más grandes que las observadas en el epitelio columnar normal. Suele ser aparente alguna tendencia al sangrado de estas papilas. Puede observarse también en áreas de erosión (erosión que puede ser levantada sobre la superficie circundante), amarillo rojiza, con márgenes no definidos, con superficie irregular que semeja cambios papilares. El blanqueamiento al acético suele ser más intenso que el observado en el epitelio columnar normal, pero más irregular del observado en los papilomas.

En otros casos la superficie del Cérvix parece un coliflor, con papilas de variados tamaños y formas, algunas confluentes. Los vasos atípicos son frecuentes así como los orificios glandulares abiertos.

\section{Manifestaciones de Zona de Transformación (ZT)}

Se observa erosión con márgenes indistintos, puede no presentarse blanqueamiento al acético, pero si son muy constantes los orificios glandulares abiertos. Los 
vasos en sauce y raíces son hallazgos frecuentes. El color amarillento de la superficie es también frecuente. Los orificios glandulares abiertos son una de las características del adenocarcinoma, pudiendo ser pequeños o de gran tamaño.

\section{Manifestaciones semejantes a tejido de granulación}

La superficie que presenta patrón de tejido de granulación es frecuente, pudiendo tener áreas de necrosis. La superficie es lisa pero irregular. Los vasos semejantes a raíces y desechos de lanitas, así como el sangrado fácil son frecuentes.

\section{Hallazgos colposcópicos del adenocarcinoma muco productor. (Secretante de exceso de moco)}

Este tipo de adenocarcinoma verdadero secreta grandes cantidades de moco, está muy bien diferenciado histológicamente.

La mayoría de mujeres que lo presentan se encuentran en la cuarta década de la vida. Suelen informar una excesiva descarga vaginal por años, sin sangre o muy poca. La citología suele ser negativa debido a la gran cantidad de moco. Este tipo de adenocarcinoma normalmente no es sospechado en el examen de rutina.

A simple vista el cérvix es turgente y elástico y la superficie erodada se encuentra cubierta por moco viscoso. Aunque el cáncer está en el área erodada, normalmente se observa ulceración, necrosis ni sangrado.

A la colposcopia la superficie es indistinta y amarillenta. Las imágenes son características de la transforma- ción papilar o indistintas, existiendo en todos los casos grandes glándulas abiertas de forma irregular. Los vasos suelen ser semejantes a raíces pero en otros casos la congestión oscurece el patrón vascular. Los hallazgos se diferencian poco de los del adenocarcinoma típico.

\section{Hallazgos colposcópicos en el carcinoma adenoescamoso de cérvix}

Se ha informado que el $48 \%$ de los adenocarcinomas de Cérvix son verdaderos mientras que el $47 \%$ son carcinomas adenoescamosos. La inspección y palpación muestra los mismos hallazgos que en el adenocarcinoma verdadero.

Colposcópicamente los hallazgos son de adenocarcinoma en las áreas de adenocarcinoma y hallazgos de Zona de Transformación Atípica en el área de carcinoma escamocelular. Sin embargo las dos áreas no suelen estar separadas claramente sino mezcladas, y en ocasiones con predominio de alguno de los dos tipos. Así que sólo en pocos casos pueden observarse simultáneamente imágenes papilares definidas, hallazgos semejantes a granulación o semejantes a zona de transformación junto a imágenes de Zona de Transformación Atípica tales como mosaico, base, epitelio acetoblanco, keratosis y vasos atípicos. En los casos avanzados los hallazgos colposcópicos suelen ser semejantes a los del adenocarcinoma típico: Sangrado, degeneración de la superficie, necrosis, por lo que la colposcopia rara vez permite diferenciar el carcinoma adenoescamoso del adenocarcinoma verdadero.

\section{BIBLIOGRAFIA}

1. González F. Cáncer del cuello uterino. Libro de Texto FLASOG. Vol. 1 Caracas, Editorial Ateproca, 1996.

2. De Palo G. Neoplasias Invasoras del Tracto Genital Inferior. Colposcopia y patología del tracto genital inferior. Ed. Médica Panamericana, Buenos Aires, segunda edición, 1996.

3. Ueki M. Cervical adenocarcinoma: A Colposcopic atlas. Ed. Ishiyaku EuroAmerica, Inc. St Louis, EE.UU. 1985.
4. ASCCP. Practice Guidelines: Management of Glandular Abnormalities in the Cervical Smear. Journal of Lower Genital Tract Disease 1997; 1(1): 41-45.

5. Creasman WT. New gynecologic cancer staging. Gynecologic Oncology 1995; 58(2): 157-158.

6. National Cancer Institute. Cáncer cervicouterino (CS-100103). http:/ /bitmed.med.uchile.cl/menuesp.html. 